Recepción: 20 / 12 / 2017

Aceptación: 15 / 02 / 2018

Publicación: 8 / 05 / 2018

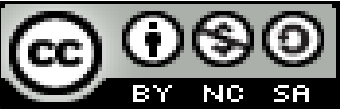

Ciencias Agrícolas

Artículo de Revisión

\title{
Calidad del suelo empleado con fines agrícolas en el Valle de Joa, Cantón Jipijapa
}

\section{Soil quality used for agricultural purposes in the Joa Valley, Jipijapa Canton}

\section{Qualidade da terra utilizada para fins agrícolas no Vale do Joa, Jipijapa (cantão)}

\author{
Alex J. Quimis-Gómez ${ }^{\mathrm{I}}$ \\ alex.quimis@unesum.edu.ec \\ Julio J. Jaramillo-Véliz II \\ julio.jaramillo@unesum.edu.ec \\ Yamel de las Mercedes Álvarez-Gutiérrez ${ }^{\text {III }}$ \\ yamel.alvarez@unesum.edu.ec \\ Aldo J. Rodríguez-Avilés IV \\ aldo.R_aviles@outlook.com
}

Correspondencia: alex.quimis@unesum.edu.ec

I Magister en Administración Ambiental; Ingeniero en Medio Ambiente; Docente de la Universidad Estatal del Sur de Manabí, Manta, Ecuador.

II. Magister en Sistemas de Información Geográfica Aplicada a la Conservación y Desarrollo Sostenible; Ingeniero Agrícola; Docente de la Universidad Estatal del Sur de Manabí, Manta, Ecuador.

III. Magister en Administración Ambiental; Licenciada en Administración de Empresas Turísticas y Hoteleras; Ingeniero en Medio Ambiente; Docente de la Universidad Estatal del Sur de Manabí, Manta, Ecuador.

IV. Estudiante de la Universidad Estatal del Sur de Manabí, Manta, Ecuador. 
Alex J. Quimis-Gómez; Julio J. Jaramillo-Véliz; Yamel de las Mercedes Álvarez-Gutiérrez; Aldo J. Rodríguez-Avilés

\section{Resumen}

La presente investigación se efectuó con la finalidad de determinar la variabilidad de los nutrimentos en los suelos, en un área destinada a cultivos de ciclo corto en la comunidad de Joa perteneciente al cantón Jipijapa, provincia de Manabí. El diseño de investigación descriptiva aplicado mediante métodos de observación, inductivo, análisis y de síntesis. Los resultados de los análisis físicos y químicos de las muestras de suelo, determinaron la potencialidad nutricional de los suelos. Las variables analizadas fueron los nutrientes de N, P, K, Ca, Mg, S, Zn, Cu, Fe, Mn, B, Mo, $\mathrm{pH}$ del suelo fueron realizados en el Laboratorio de Suelos, Tejidos. Vegetales y Aguas del INIAP Estación Experimental Pichilingue. Los resultados fueron un nivel óptimo en la mayoría de nutrientes, no así en nitrógeno que fue bajo. La textura de los suelos oscila entre franco arcilloso a franco limoso y un pH entre neutro y alcalino (7-8). El diagnóstico señaló que los agricultores no aplican prácticas agrícolas de conservación de los suelos y que sus labores empleadas están relacionadas con una agricultura convencional. Adicionalmente, desconocen la importancia de las prácticas conservacionistas de los suelos, ya que no han recibido capacitaciones sobre esta problemática. Se recomienda, que los productores reciban capacitación sobre técnicas conservacionistas de los suelos, que se realicen nuevas investigaciones en áreas con condiciones similares, para verificar los resultados encontrados y proponer alternativas para remediar y conservar los recursos naturales como son el agua y suelo.

Palabras clave: Suelos, agrícolas, contaminación, análisis. 


\begin{abstract}
The present investigation was carried out with the purpose of determining the variability of the nutrients in the soils, in an area destined to short cycle crops in the community of Joa belonging to the canton Jipijapa, province of Manabí. The design of descriptive research applied by methods of observation, induction, analysis and synthesis. The results of the physical and chemical analyzes of the soil samples determined the nutritional potential of the soils. The variables analyzed were the nutrients of N, P, K, Ca, Mg, S, Zn, Cu, Fe, Mn, B, Mo, pH of the soil were made in the Soil, Tissue Laboratory. Vegetables and Waters of the INIAP - Pichilingue Experimental Station. The results were an optimum level in most nutrients, but not in nitrogen, which was low. The texture of the soils ranges from clay loam to loamy loam and a $\mathrm{pH}$ between neutral and alkaline (7-8). The diagnosis indicated that farmers do not apply agricultural practices for soil conservation and that their work is related to conventional agriculture. Additionally, they are unaware of the importance of soil conservation practices, since they have not received training on this problem. It is recommended that producers receive training on soil conservation techniques, that new research be conducted in areas with similar conditions, to verify the results found and propose alternatives to remedy and conserve natural resources such as water and soil.
\end{abstract}

Keywords: Soils, agricultural, pollution, analysis. 
Alex J. Quimis-Gómez; Julio J. Jaramillo-Véliz; Yamel de las Mercedes Álvarez-Gutiérrez; Aldo J. Rodríguez-Avilés

\section{Introducción.}

Hoy en día en una agricultura altamente desarrollada, es necesario disponer de múltiples recursos tanto naturales como tecnológicos y uno de esos, es el recurso natural suelo. Rattan Lal en su libro "Soil Quality and Agricultural Sustainability", define a la calidad del suelo como un conjunto de capacidades del mismo para realizar funciones específicas y en términos de agricultura lo define como su capacidad para lograr producciones sostenibles, mencionando además que existe un fuerte vínculo entre la calidad de los suelos y la agricultura sostenible (Lal,1998:3).

Los suelos que cuentan con una máxima calidad, tienen la capacidad de ser altamente productivos y provocar un mínimo deterioro al ambiente (Moreno et al., 2015, pág. 34) por lo que es necesario conocer la calidad de los suelos, ya que mediante el conocimiento de la calidad de los mismos, se puede potenciar los resultados agrícolas y por supuesto hacer sostenible los variados sistemas de manejo empleados.

Según Abi \& Arrieche, (2012) existe una relación entre la calidad del suelo y las prácticas agrícolas, señalando que diversas prácticas agrícolas como la disminución de insumos químicos, rotación, manejo integrado de plagas, entre otras, contribuyen a una buena calidad del suelo.

El suelo al estar compuesto por agua, aire, materia orgánica e inorgánica, organismos vivos y por partículas minerales, en él se lleva a cabo una de las actividades económicas más antiguas que es la agricultura. Es considerado un suelo agrícola a aquel que se lo usa en el ámbito de la productividad, apto para todo tipo de cultivo, su capa fértil se forma a lo largo del tiempo, pero por malas prácticas esta se puede perder en poco tiempo. Su conservación es muy importante para mantener su productividad, porque cuando la capa superior se pierde existe menos retención de agua 
y las raíces ya no tienen soporte, se pierde la materia orgánica, el nitrógeno, el fósforo, nutrientes y otros elementos (Tamayo \& Lizbeth, 2016:88).

Los conceptos de la calidad del suelo consideran la capacidad para funcionar dentro de los límites del ecosistema para sustentar a la productividad biológica, mantener la calidad ambiental y promover la salud de las plantas, animales y cabe decir su asociación al desarrollo agrícola sostenible que concilia la protección de la naturaleza y mejora el bienestar humano a través del tiempo (Bogado, 2013:1).

Ecuador, un país eminentemente agrícola, produce una diversidad de especies vegetales de alta calidad, tanto para consumo nacional o exportar, para lo cual hace uso de una diversidad de suelos, sin importar una determinada calidad, sin embargo, muchos cultivos son muy exigentes en cuanto a la calidad física, química y biológica de los suelos. Criterios que aún son muy lejanos para cumplir bajo los sistemas agrícolas de poca tecnología empleados en el país.

Manabí, es una provincia con vocación agrícola diversa, desde especies hortícolas, de ciclo perennes hasta especies maderables. También, dispone de una diversidad de suelos y variada ecología. No obstante, sus sistemas de producción empleados, son tradicionalistas, no requieren de técnicas agrícolas sofisticadas, mucho menos de exigentes requerimientos en cuanto a la calidad de recursos naturales tales como suelo y agua. Jipijapa, es un cantón ubicado al sur de la provincia, se ha caracterizado por su vocación agrícola, especialmente en cultivos como café, que se produce en las zonas con mayor precipitación y maíz en las zonas bajas, en donde las precipitaciones son muy escasas, el éxito de la actividad agrícola en esta zona es incierto.

Karlen, Eash, \& Unger en su estudio "Soil and Crop Management Effects on Soil Quality Indicators". Determinaron que existe interacción entre las actividades humanas y los factores 
Alex J. Quimis-Gómez; Julio J. Jaramillo-Véliz; Yamel de las Mercedes Álvarez-Gutiérrez; Aldo J. Rodríguez-Avilés

naturales que influyen en la calidad del suelo. Quienes recomiendan el empleo de prácticas como: labranza mínima, rotación de cultivos y cultivos de cobertura, las cuales favorecen a la calidad del suelo, no obstante, concluyen que es el carbono el factor crítico para una buena calidad del suelo por lo que es necesario incorporar la materia orgánica. (Karlen, Eash, \& Unger, 1992:7)

La calidad del suelo también se referencia a la capacidad que presenta un suelo específico para funcionar dentro de los límites de los ecosistemas ya sean naturales o antrópicos, asegurando así la productividad. Siendo capaz de desempeñar dentro de este, manteniendo la calidad del agua, el ciclaje de nutrientes y la producción de la materia orgánica animal y vegetal (Loor \& Zambrano, 2016:6).

En los últimos años, una comuna del cantón que ha cobrado importancia en la actividad agrícola, principalmente, por que hace uso de las aguas residuales, provenientes de la planta de tratamiento de aguas servidas. Actualmente, unas 80 familias se dedican a realizar actividades agrícolas durante el verano, producen una diversidad de productos que son comercializados en el mercado local, sin embargo, se desconoce la calidad e inocuidad de los productos comercializados. Así, como, los suelos pueden ser afectados por el empleo de las aguas residuales, que pueden contener cierta cantidad de productos no deseables que alteren la calidad física, química y biológica de los suelos del valle de la comuna Joa.

\section{Materiales y métodos.}

El tipo de estudio es descriptivo, Se utilizó en esta investigación las técnicas de muestreo de los suelos, análisis físico y químico de suelo, encuesta y la entrevista. Los métodos que fueron utilizados fueron: observación, inductivo, análisis y de síntesis. La técnica utilizada fue de muestreó 
de suelos para el análisis físico y químico respectivo y la encuesta para recoger la información mediante un formulario técnicamente elaborado.

\section{Resultados.}

Tabla 1. Resultados de análisis físico-químico de los suelos del lote Andil.

\begin{tabular}{|c|c|c|c|c|c|}
\hline \multirow[b]{2}{*}{ Parámetros } & \multirow[b]{2}{*}{$\begin{array}{l}\text { Valores } \\
\text { de referencia } \\
\text { INIAP }\end{array}$} & \multicolumn{3}{|c|}{ Fechas de Muestreo/agricultor } & \multirow[b]{2}{*}{ Promedio } \\
\hline & & $\begin{array}{l}\text { Lote 1, Andil } \\
16 \text { noviembre } \\
2016\end{array}$ & $\begin{array}{c}\text { Lote 1, Andil } \\
17 \text { marzo } \\
2017\end{array}$ & $\begin{array}{c}\text { Lote 1, } \\
\text { Andil } \\
17 \text { junio } 2017\end{array}$ & \\
\hline pH & $6.0-7.5$ & 5,2 & 7,0 & 7,2 & 6,47 \\
\hline $\mathbf{N}$ & 40 ppm & 43,0 & 21,0 & 34,0 & 32,67 \\
\hline $\mathbf{P}$ & 14 ppm & 198,0 & 143,0 & 54,0 & 131,67 \\
\hline $\mathbf{K}$ & $0.38 \mathrm{meq} / 100 \mathrm{ml}$ & 1,20 & 3,63 & 2,39 & 2,41 \\
\hline $\mathbf{C a}$ & $8.9 \mathrm{meq} / 100 \mathrm{ml}$ & 21,0 & 16,0 & 25,0 & 20,67 \\
\hline Mg & $2.3 \mathrm{meq} / 100 \mathrm{ml}$ & 5,7 & 7,7 & 5,2 & 6,20 \\
\hline $\mathbf{S}$ & 20 ppm & 6,0 & 8,0 & 16,0 & 10,00 \\
\hline $\mathbf{Z n}$ & 7 ppm & 5,9 & 3,0 & 3,1 & 4,00 \\
\hline $\mathbf{C u}$ & 4 ppm & 8,6 & 7,1 & 5,4 & 7,03 \\
\hline Fe & 40 ppm & 164 & 56 & 51 & 90,33 \\
\hline Mn & 15 ppm & 7,2 & 5,4 & 5,5 & 6,03 \\
\hline B & 0,40 ppm & 0,24 & 0,82 & 1,13 & 0,73 \\
\hline MO & $>5 \%$ & 4,1 & 0,7 & 0,9 & 1,90 \\
\hline $\mathrm{Ca} / \mathrm{Mg}$ & $2.6-8.0$ & 3,6 & 2,0 & 4,8 & 3,47 \\
\hline $\mathrm{Mg} / \mathrm{K}$ & $7.5-15$ & 4,75 & 2,12 & 1,77 & 2,88 \\
\hline $\mathrm{Ca}+\mathrm{Mg} / \mathrm{K}$ & $27.5-55.0$ & 22,25 & 6,53 & 10,31 & 13,03 \\
\hline \multicolumn{2}{|c|}{ Textura } & $\begin{array}{l}\text { Franco- } \\
\text { arcilloso }\end{array}$ & $\begin{array}{l}\text { Franco- } \\
\text { arcilloso }\end{array}$ & Franco & \\
\hline \multicolumn{6}{|l|}{ ALTO } \\
\hline \multicolumn{6}{|l|}{ BAJO } \\
\hline
\end{tabular}

Fuente: Laboratorio de Suelos, Tejidos. Vegetales y Aguas del INIAP - Estación Experimental Pichilingue.

\section{Elaboración Propia.}


Alex J. Quimis-Gómez; Julio J. Jaramillo-Véliz; Yamel de las Mercedes Álvarez-Gutiérrez; Aldo J. Rodríguez-Avilés

\section{Análisis interpretación de los resultados}

En esta tabla $\mathrm{N}^{\circ} 1$, se presenta un resumen de las cinco localidades incluyendo la localidad de Andil, tomada como un lugar de referencia, para comparar con las localidades de Joa, en la indicada tabla, se observan los resultados de los análisis físico y químico del suelo de las muestras que fueron tomadas en tres fechas diferentes (noviembre 16 del 2016, marzo 17 del 2017 y junio 17 del 2017). Se consideraron los parámetros más importantes que puedan demostrar alguna diferencia en la calidad de los suelos. Los parámetros físicos, textura fue evaluada, señalando que en las localidades Andil y del Sr. Darío León Lucas (sitio Paneles Solares) son suelos de textura franco arcillosos, mientras tanto en las localidades Finca San Luis del productor Sr. Stalin Quimís, Nawi y Cruz de Hachi del productor Sr. Erwin Facundo la textura de los suelos es franco limoso. En cuanto al pH, se encontró que en el lote 1, Andil son suelos ligeramente ácidos, su valor fue de 6,47 mientras en las demás localidades, estos valores fluctúan desde ligeramente alcalino $(7,53)$ a suelos alcalino $(8,10)$ considerados suelos alcalinos. En cuanto a los parámetros nutricionales, estos señalan bajos contenidos de N, Zn, Fe, Mn y MO, solo en el sitio Andil, el Fe, presenta un nivel alto. Altos valores, se observan en los parámetros nutricionales de $\mathrm{P}, \mathrm{K}, \mathrm{Ca}, \mathrm{Mg}, \mathrm{S}, \mathrm{Cu}$ y B. Las relaciones $\mathrm{Ca} / \mathrm{Mg}$ se encuentran adecuadas en la mayoría de las localidades muestreadas salvo en la de Paneles Solares. Mientras tanto en las relaciones, $\mathrm{Mg} / \mathrm{K} ; \mathrm{Ca}+\mathrm{Mg} / \mathrm{K}$ estuvieron por debajo del nivel mínimo del rango adecuado. La información obtenida confirma, lo conocido, que la mayoría de suelos de Manabí, son bajos en $\mathrm{N}$ y MO mientras son altos $\mathrm{P}, \mathrm{K}, \mathrm{Ca}$. Mientras otras variable $\mathrm{pH}$ y textura no son una limitante para el desarrollo de la agricultura o en todo caso, la calidad del suelo no está siendo afectada significativamente. 
Tabla $N^{o}$ 2: Correspondiente a implementos utilizados para la preparación de suelos.

\section{Alternativas}

Tipo de enganche, motocultor

Tipo de tracción, animal

Tipo de labor, arados

Tipo de profundidad de trabajo, arado de cincel

\section{Frecuencias}

10

0

0

5

\section{Porcentajes}

80

0

0

20

100

Fuente: Información de los habitantes de la comunidad del valle Joa del cantón Jipijapa.

\section{Elaboración propia.}

\section{Análisis interpretación de los resultados}

Esta tabla $\mathrm{N}^{\mathrm{o}} 2$ se demuestra que el $80 \%$ de los encuestados ha usado implementos tipo de enganche (motocultor), mientras que un $20 \%$ ha usado clasificadas por la profundidad de trabajo (arados), esto muestra una tendencia de los agricultores a usar tecnologías de gran potencia, lo que repercute directamente en los cambios de las propiedades físicas de los suelos, que de no aplicar medidas de conservación en los suelos a largo plazo podría haber algún cambio significativo en la calidad de los suelos. 
Alex J. Quimis-Gómez; Julio J. Jaramillo-Véliz; Yamel de las Mercedes Álvarez-Gutiérrez; Aldo J. Rodríguez-Avilés

Tabla $N^{o}$ 3: Correspondiente al análisis físico-químico de suelo

\begin{tabular}{lcc}
\hline Alternativa & Frecuencia & Porcentaje \\
\hline $\mathrm{Si}$ & 0 & 0 \\
$\mathrm{No}$ & 15 & 100 \\
\hline Total & 15 & 100
\end{tabular}

Fuente: Información de los habitantes de la comunidad del valle Joa del cantón Jipijapa.

\section{Elaboración propia.}

\section{Análisis interpretación de los resultados.}

En la tabla $\mathrm{N}^{\mathrm{o}} 3$, se puede observar que el $100 \%$ de los encuestados indicó que no conoce sobre lo que significa realizar análisis de suelos. Por lo que se cree que los agricultores de la comunidad aplican los insumos fertilizantes sin tener en cuenta las necesidades reales de cada suelo. Lo que posiblemente puede afectar en determinados momentos la calidad química de los suelos.

Tabla $N^{o}$ 4: Correspondiente al tipo de cultivo de siembra.

\begin{tabular}{|c|c|c|}
\hline Alternativas & Frecuencias & Porcentajes \\
\hline Maíz en invierno, pimiento & 15 & 100 \\
\hline Tomate, cebolla en verano & & \\
\hline
\end{tabular}

Fuente: Información de los habitantes de la comunidad del valle Joa del cantón Jipijapa.

\section{Elaboración propia.}




\section{Análisis interpretación de los resultados}

Como se puede apreciar en la tabla $\mathrm{N}^{\circ} 4$, el $100 \%$ de los encuestados siembra preferentemente en invierno maíz y en verano, hortalizas tales como pimiento, tomate, cebolla etc. La tendencia de los resultados indica que estos suelos son usados intensivamente, lo que puede a corto o mediano plazo disminuir su calidad nutritiva, lo cual afectaría también su calidad nutricional.

Tabla $N^{o}$ 5: Correspondiente a la labranza conservacionista.

\begin{tabular}{lcc}
\hline Alternativa & Frecuencia & Porcentaje \\
\hline $\mathrm{Si}$ & 0 & 0 \\
$\mathrm{No}$ & 15 & 100 \\
\hline Total & 15 & 100
\end{tabular}

Fuente: Información de los habitantes de la comunidad del valle Joa del cantón Jipijapa.

\section{Elaboración propia.}

\section{Análisis interpretación de los resultados}

En la tabla №5, se observa que el 100\% de los encuestados indicó que no usa técnica de labranza conservacionista. Resultados que están relacionados a empleo de labranzas convencionales y que a mediano o largo plazo pueden estar incidiendo en la calidad de los suelos de este sitio agrícola del cantón. 
Alex J. Quimis-Gómez; Julio J. Jaramillo-Véliz; Yamel de las Mercedes Álvarez-Gutiérrez; Aldo J. Rodríguez-Avilés

Tabla $N^{o}$ 6: Correspondiente a métodos de conservación para el suelo.

\begin{tabular}{lcc}
\hline & & \\
Alternativa & Frecuencia & Porcentaje \\
\hline $\mathrm{Si}$ & 3 & 20 \\
$\mathrm{No}$ & 12 & 80 \\
\hline Total & 15 & 100
\end{tabular}

Fuente: Información de los habitantes de la comunidad del valle Joa del cantón Jipijapa.

\section{Elaboración propia.}

\section{Análisis interpretación de los resultados}

En la tabla $\mathrm{N}^{\circ} 6$ se observan los resultados de la pregunta relacionada sobre aplicación de métodos de conservación de los suelos. El 80\% de los agricultores encuestados indicó que no aplica métodos de conservación para los suelos, y tan solo un $20 \%$ respondió que aplica métodos que ayudan a la conservación de los suelos. Siendo la sustitución de los abonos inorgánicos la única técnica de conservación de los suelos empleada. Lo que posiblemente, de no existir la incorporación de alternativas de métodos de conservación de los suelos estos pueden ser afectados en su calidad. 
Tabla $N^{o}$ 7: Correspondiente a la capacitación en manejo adecuado del suelo

\begin{tabular}{|c|c|c|}
\hline Alternativa & Frecuencia & Porcentaje \\
\hline $\mathrm{Si}$ & 3 & 20 \\
\hline No & 12 & 80 \\
\hline Total & 15 & 100 \\
\hline
\end{tabular}

Fuente: Información de los habitantes de la comunidad del valle Joa del cantón Jipijapa.

\section{Elaboración propia.}

\section{Análisis interpretación de los resultados}

En la tabla $\mathrm{N}^{\mathrm{o}} 7$ se muestran los resultados, correspondiente a la pregunta si los productores han recibido capacitaciones, el $80 \%$ de los agricultores encuestados indicó que no han recibido capacitaciones sobre esta problemática y tan solo un $20 \%$ mencionó que sí. El conocimiento es importante, ya que permite tomar decisiones, a la hora de seleccionar alternativas de manejo de técnicas conservacionistas de suelos. Lamentablemente, no existen políticas gubernamentales que trabajen en estos temas que no son comunes.

\section{Conclusiones.}

Mediante los análisis físicos y químicos de las muestras de suelo, se pudo determinar la potencialidad nutricional de los suelos y su calidad física, e indicó que existe un buen suministro de la mayoría de nutrientes, sin embargo, se encontró bajo nivel de Nitrógeno, principalmente. 
Alex J. Quimis-Gómez; Julio J. Jaramillo-Véliz; Yamel de las Mercedes Álvarez-Gutiérrez; Aldo J. Rodríguez-Avilés

La textura de los suelos mayoritariamente se encuentran entre franco arcilloso y franco limoso, con un $\mathrm{pH}$ entre neutro (7) y alcalino (8), los que son ideales para el desarrollo de cultivos de ciclo corto como perenne en la comunidad en estudio.

Se evidencio que los agricultores de la comunidad de Joa en su mayoría no utilizan prácticas agrícolas de conservación de los suelos y que sus labores actualmente están relacionadas con una agricultura convencional que desagradan los recursos naturales presentes y futuros.

Los agricultores desconocen la importancia de las prácticas conservacionistas de los suelos y no han recibido capacitaciones sobre esta problemática. Además las prácticas agrícolas aplicadas, pueden afectar la calidad de los suelos a corto o mediano plazo, de no aplicar alternativas conservacionistas.

\section{Recomendaciones.}

De acuerdo a las conclusiones obtenidas se establecen las siguientes recomendaciones, que los productores del valle de la comuna de Joa, reciban capacitación en técnicas conservacionistas de los suelos, para evitar que se continúe aplicando labores agrícolas convencionales que afecten la calidad de estos recursos naturales presentes a corto y mediano plazo.

Que se efectué rotaciones de cultivos y que sean fijadores de nitrógeno para suplir las deficiencias encontradas durante el estudio.

Se recomienda que se realicen nuevas investigaciones bajo las dos épocas lluviosa y seca para verificar los resultados encontrados y proponer alternativas para remediar, si existieran daños ambientales al recurso natural como el suelo. 


\section{Bibliografía.}

Abi, R., \& Arrieche, S. (2012). Evaluación de la calidad del suelo, en el sistema Productivo orgánico la estancia, madrid, cundinamarca, 2012. Utilizando indicadores de calidad de suelos. Trabajo de grado, pontificia universidad javeriana, facultad de estudios ambientales y rurales, bogota. Recuperado el 06 de 11 de 2017.

Bogado, K. (2013). Calidad del suelo en diferentes sistemas de manejo utilizando algunos indicadores biologicos. Tesis de grado, Universidad Nacional de Asunción, Facultad de Ciencias Agrarias, San Lorenzo. Recuperado el 28 de 07 de 2017, de http://www.conacyt.gov.py/sites/default/files/TES-BN-018.pdf

Karlen, D., Eash, N., \& Unger, P. (1992). Soil and Crop Management Effects on Soil Quality Indicators. American Journal of Alternative Agriculture, 7.

Tamayo, B., \& Lizbeth, C. (2016). Calidad de agua y suelo en la cuenca del rio Ambi, para uso agricola en los campos experimentales de la ciudad del conocimiento Yachay mediante analisis fisico-quimico. Tesis de Grado, ESPE, Departamento de ciencias de la tierra y la construccion, Sangolqui. Recuperado el 16 de 11 de 2017.

Lal, R. (1998). Soil quality and agricultural sustainability. (E. R. Lal, Ed.) Soil quality and agricultural sustainability, 3 .

Loor, R., \& Zambrano, P. (2016). El cultivo de plátano (Mussa balbisiana) y la calidad. Tesis de Grado, Escuela Superior Politécnica Agropecuaria De Manabí Manuel Félix López, CARRERA Ingeniería Ambiental, Calceta.

Moreno et al. (2015). Influencia del manejo sobre la calidad del suelo. Revista Científica Ecuatoriana, 2(1), 34. 